\title{
Administrator Perspectives on ICU-to-Ward Transfers and Content Contained in Existing Transfer Tools: a Cross-sectional Survey
}

\author{
Jamie M. Boyd, $B A^{7}$, Derek J. Roberts, MD, PhD'², Jeanna Parsons Leigh, $P h D^{3}$, and \\ Henry Thomas Stelfox, MD, PhD ${ }^{4}$
}

${ }^{1}$ Departments of Critical Care Medicine and Community Health Sciences, University of Calgary, Calgary, Alberta, Canada; ${ }^{2}$ Department of Surgery, University of Calgary and the Foothills Medical Centre, Calgary, Alberta, Canada; ' ${ }^{3}$ epartments of Critical Care Medicine, O’Brien Institute for Public Health, University of Calgary and Alberta Health Services, Calgary, Alberta, Canada; ${ }^{4}$ Departments of Critical Care Medicine, Medicine and Community Health Sciences, O'Brien Institute for Public Health, University of Calgary and Alberta Health Services - Calgary Zone, Calgary, Alberta, Canada.

BACKGROUND: The transfer of critically ill patients from the intensive care unit (ICU) to hospital ward is challenging. Shortcomings in the delivery of care for patients transferred from the ICU have been associated with higher healthcare costs and poor satisfaction with care. Little is known about how hospital ward providers, who accept care of these patients, perceive current transfer practices nor which aspects of transfer they perceive as needing improvement.

OBJECTIVE: To compare ICU and ward administrator perspectives regarding ICU-to-ward transfer practices and evaluate the content of transfer tools.

DESIGN: Cross-sectional survey design.

PARTICIPANTS: We administered a survey to 128 medical and/or surgical ICU and 256 ward administrators to obtain institutional perspectives on ICU transfer practices. We performed qualitative content analysis on ICU transfer tools received from respondents.

KEY RESULTS: In total, 108 (77\%) ICU and 160 (63\%) ward administrators responded to the survey. The ICU attending physician was reported to be "primarily responsible" for the safety (93\% vs. 91\%; $p=0.515)$ of patient transfers. ICU administrators more commonly perceived discharge summaries to be routinely included in patient transfers than ward administrators $(81 \%$ vs. $60 \% ; p=0.006)$. Both groups identified information provided to patients/families, patient/family participation during transfer, and ICU-ward collaboration as opportunities for improvement. A minority of hospitals used ICUto-ward transfer tools ( $11 \%$ ) of which most ( $n=21$ unique) were designed to communicate patient information between providers $(71 \%)$ and comprised six categories of information: demographics, patient clinical course, corrective aids, mobility at discharge, review of systems, and documentation of transfer procedures.

CONCLUSION: ICU and ward administrators have similar perspectives of transfer practices and identified patient/

Electronic supplementary material The online version of this article (https://doi.org/10.1007/s11606-018-4590-8) contains supplementary material, which is available to authorized users.

Received November 6, 2017

Revised April 20, 2018

Accepted July 10, 2018

Published online July 26, 2018 family engagement and communication as priorities for improvement. Key information categories exist.

KEY WORDS: intensive care units; continuity of care; care transitions; survey research; qualitative analysis.

J Gen Intern Med 33(10):1738-45

DOI: $10.1007 / \mathrm{s} 11606-018-4590-8$

(c) Society of General Internal Medicine 2018

\section{INTRODUCTION}

The transfer of critically ill patients from the intensive care unit (ICU) to hospital ward is challenging. The ICU provides specialized, life-sustaining care to the sickest patients in the hospital. ${ }^{1,2}$ These patients have complex medical problems and reduced physiological capacity, ${ }^{3}$ leaving them susceptible to medical errors and adverse events ${ }^{4-7}$ during transitions of care.

The optimal model for ICU transfer has not been identified and pitfalls are common. ${ }^{6,8}$ Multiple factors plague these processes, including variation in practices (both within and between hospitals), communication breakdowns, capacity strains, and limitations in available resources. ${ }^{6,8-14}$ Shortcomings in the delivery of care ${ }^{9,15}$ for patients transferred from the ICU have been associated with higher healthcare costs and poor satisfaction with care. $^{16-19}$

Little is known about how hospital ward providers, who accept care of these patients, perceive current transfer practices nor which aspects of transfer they perceive as needing improvement. $^{20,21}$ To address this knowledge gap, we took advantage of a pre-existing national cross-sectional survey of Canadian ICU administrators' perspectives on ICU transfer practices ${ }^{8}$ to identify ward administrators whose units accept patients transferred from the ICUs studied and explore their perspectives regarding transfer practices. We sought to compare the perspectives of ICU and ward administrators to identify similarities and differences in perspectives of ICU transfer practices, and to evaluate the content underlying the transfer tools used to facilitate 
ICU-to-ward transfer processes to catalog the range of information elements considered essential to transfer communication.

\section{METHODS}

\section{Research Approach}

This study was part of a multi-study program of work to reengineer the transition of patients from ICU to ward to improve patient safety, continuity of care, and quality of care. ${ }^{6}$, 8, 18, 22 Building on our previous study of ICU stakeholder perspectives on patient care transfer practices, ${ }^{8}$ we conducted a national cross-sectional survey of Canadian medical and surgical ward administrators (i.e., directors, unit managers) whose hospital wards routinely accept patients in transfer from the previously studied ICUs. Administrators were asked their perspectives on current transfer practices and any tools used to facilitate the transfer of ICU patients onto their ward. Ethics approval was obtained from the Conjoint Health Research Ethics Board at the University of Calgary (EID-24282).

Study Participants. In our previous study, we administered surveys to 128 administrators representing 128 adult medical-surgical ICUs ( $n=128$ hospitals) in Canada to solicit their perspectives on ICU transfer practices. ${ }^{8}$ For this project, we employed a snowball sampling strategy where ICU administrator participants from our previous study were asked to identify an administrator for one medical ward and one surgical ward at their institution that routinely accept patients in transfer from ICU (i.e., $n=2$ ward administrators for each ICU and hospital for a total of 256 ward administrators). Ward administrators were identified from a search of the hospitals' websites, contacts of the project team, and local telephone directories. To ensure feasibility, we restricted the study participants to administrators in Canadian hospitals who could provide a unit-level perspective on the patient transfer process from ICU. We did not include physician and nurse front line providers in our survey sample (although some administrators also serve as frontline providers) because we had explored their perspectives in our earlier study. ${ }^{8}$

Survey Design and Testing. Development of the ICU administrator survey instrument is described elsewhere. ${ }^{8}$ For the ward administrator survey, the ICU survey was modified to reflect the perspective of a hospital ward that would receive patients in transfer from ICU and respondents were asked to provide a unit-level perspective on the process of receiving a patient transferred from ICU. The final survey was comprised of 27 close-ended questions and 2 open-ended questions (see Appendix 1 [available online]). Both ICU and ward administrator surveys invited participants to share any tools used to facilitate the transfer process.
Survey Administration. The ICU administrator survey was distributed from February 23, 2012, to July $31,2012 .^{8}$ After using the ICU administrator survey data to identify wards that receive patients transferred from the ICU and looking up the contact information, the ward administrator survey was distributed from June 10, 2013, to August 31, 2013. Ward administrators were contacted via telephone or email (based on contact information available) and offered participation. Three follow-up phone calls or emails were sent, in 3-week intervals, to non-responders.

Content analysis. We performed a qualitative content analysis on the transfer tools received from both ICU and ward administrators to identify common information elements (i.e., items of patient information or tasks requiring completion). Content analysis is a validated method to synthesize various types of information. ${ }^{23,}{ }^{24}$ We defined a tool as an instrument that details the essential steps needed to complete a task (e.g., checklist), requires providers to input patient data (e.g., transfer summary), or is designed to communicate pertinent information about the transfer to patients/families (e.g., patient/family information brochure) ${ }^{25-28}$ We selected tools for the content analysis using the following criteria: (1) purpose of the tool is to facilitate the transfer of patient care between the ICU and ward, (2) the tool was designed to be used for individual patients (e.g., we excluded policies designed to inform care of patient populations), and (3) the tool was used by a member of the patient's healthcare team (ICU or ward). Tools that reported unit-level data (e.g., workflow report) were administrative documents (e.g., transfer orders, clinical practice guidelines) or not related to patient transfer to ward (e.g., tools to facilitate hospital transfer to community) were excluded.

Transfer Tool Data Extraction. A qualitative content analysis approach was used to develop a codebook for codes (i.e., unique types of information or "information elements" contained within the transfer tools) that emerged during analysis. ${ }^{24,}{ }^{29}$ Two investigators (JMB, DJR) independently, and in duplicate, sorted the individual information elements into groups with other similar elements. The investigators utilized latent coding ${ }^{29}$ to develop codes to describe the individual information elements (sub-codes) within each grouping and discussed the codes (sub-codes) iteratively until consensus was reached. ${ }^{30-32}$ Two investigators (JMB, DJR) used an extraction form based on the codebook to code each decision-support tool independently and in duplicate (see Appendix 2 [available online] for list of data extraction elements).

Statistical Analysis. The strategy for analysis of both the survey results and content analysis was descriptive. Survey responses for both ICU and ward administrators were summarized using proportions and compared using chi- 
squared and Fisher's exact tests. For the content analysis of transfer tools, counts and percentages were reported for the identified codes and sub-codes. Statistical analyses were performed using Stata SE version 13.1 (Stata Corp, College Station, TX).

\section{RESULTS}

Of the 128 surveys sent to ICU administrators ${ }^{8}$ and 256 surveys sent to ward administrators ( $n=128$ hospitals), 108 $(84 \%)^{8}$ and $160(63 \%)$ administrators responded, respectively. Response rates were similar for ward administrators of medical, surgical, and combined medical/surgical units.

Table 1 Characteristics of Survey Participants

\begin{tabular}{|c|c|c|}
\hline$\overline{\text { Characteristics }}$ & $\begin{array}{l}\text { ICU (\%) } \\
(n=108)\end{array}$ & $\begin{array}{l}\text { Ward (\%) } \\
(n=160)\end{array}$ \\
\hline \multicolumn{3}{|l|}{ Role within ICU/receiving ward } \\
\hline Manager & $71(68)$ & $96(60)$ \\
\hline Nurse & $11(10)$ & $28(18)$ \\
\hline Director & $22(21)$ & $29(18)$ \\
\hline Physician & $1(1)$ & $6(4)$ \\
\hline \multicolumn{3}{|l|}{ Academic status } \\
\hline Teaching setting & $79(73)$ & $108(68)$ \\
\hline Non-teaching setting & $29(27)$ & $52(32)$ \\
\hline \multicolumn{3}{|l|}{ Geographic location } \\
\hline Urban & $96(89)$ & $147(92)$ \\
\hline Rural & $12(11)$ & $13(8)$ \\
\hline \multicolumn{3}{|l|}{ Number of operational beds } \\
\hline$<10$ & $29(30)$ & $1(1)$ \\
\hline $10-20$ & $43(45)$ & $11(7)$ \\
\hline$>20$ & $24(25)$ & $139(92)$ \\
\hline \multicolumn{3}{|c|}{ Average number of discharges to ward/admissions from ICU per week } \\
\hline$<10$ & $37(39)$ & $125(90)$ \\
\hline $10-25$ & $53(55)$ & $10(7)$ \\
\hline$>25$ & $6(6)$ & $4(3)$ \\
\hline \multicolumn{3}{|l|}{ Nurse-to-patient ratio } \\
\hline $1: 1$ or $1: 2$ & $102(94)$ & $6(4)$ \\
\hline $1: 3$ or $1: 4$ & $6(6)$ & $62(40)$ \\
\hline $1: 5$ or $1: 6$ & $0(0)$ & $75(48)$ \\
\hline $1: 7$ or $1: 8$ & $0(0)$ & $4(3)$ \\
\hline $1: 9$ or $1: 10$ & $0(0)$ & $6(4)$ \\
\hline $1:>10$ & $0(0)$ & $3(2)$ \\
\hline \multicolumn{3}{|l|}{ Patient population includes* } \\
\hline Surgical & $100(93)$ & $87(54)$ \\
\hline Trauma & $45(42)$ & $30(19)$ \\
\hline Medical & $102(94)$ & $116(73)$ \\
\hline Neuroscience & $51(47)$ & $29(18)$ \\
\hline Burns & $20(19)$ & $11(7)$ \\
\hline ICU offers ventilation support & $106(100)$ & - \\
\hline Intensivist led ICU rounds & $83(81.4)$ & _- \\
\hline \multicolumn{3}{|c|}{$\begin{array}{l}\text { Multidisciplinary providers participating in daily patient rounds } \\
\text { include },+\end{array}$} \\
\hline Registered nurse & $97(96)$ & $158(100)$ \\
\hline Attending physician & $94(93)$ & $142(90)$ \\
\hline Respiratory therapist & $92(91)$ & $41(26)$ \\
\hline Pharmacist & $84(83)$ & $93(59)$ \\
\hline Dietician & $76(75)$ & $59(37)$ \\
\hline Resident & $65(64)$ & $88(56)$ \\
\hline Social worker & $41(41)$ & $62(39)$ \\
\hline Fellow & $39(39)$ & $30(19)$ \\
\hline Occupational therapist & $23(23)$ & $67(42)$ \\
\hline Nurse manager & $21(21)$ & $17(11)$ \\
\hline
\end{tabular}

Data are expressed as number (percentage) unless otherwise indicated Response rates for individual questions varied from 94 to $100 \%$ (median $100 \%$, interquartile range 97 to $100 \%$ )

*Responses are not mutually exclusive

${ }^{\dagger}$ ICU responses represent participation in daily patient care rounds whereas ward responses represent participation in daily patient care

\section{Survey Results}

Respondent Characteristics. The characteristics of the survey participants and their institutions are summarized in Table 1. Administrators were primarily managers (e.g., unit manager, patient care manager) (60\%) who worked at teaching hospitals (68\%). Compared to ICU administrators, ward administrators reported a higher number of operational beds (median 10-20 beds vs. $>20$ beds respectively) and a higher nurse-to-patient ratio (median $1: 1$ or $1: 2$ vs. $1: 5$ or $1: 6$ ).

Transfer Procedures. Administrator perspectives of current ICU transfer practices are outlined in Supplementary Table 1 (available online). Responses between both groups were similar with a few notable exceptions. ICU administrators were more likely than ward administrators to report that transfers of care included formalized nurse-to-nurse communication ( $84 \%$ vs. $68 \%, p=0.022)$, that the telephone was the most frequent mode of communication $(75 \%$ vs. $61 \%, p=$ 0.018 ), and that patient summaries are included during the transfer $(81 \%$ vs. $60 \%, p=0.006)$.

Communication with Patients and Families. Supplementary Table 2 (available online) outlines ICU and ward administrators' perceptions of how their units provide information to patients and families during the transfer process. Both groups reported frequently communicating verbally ( $99 \%$ vs. $100 \%, p=0.239$ ), but infrequently in writing ( $26 \%$ vs. $20 \%, p=0.224$ ), with patients and their family members during the transfer process. Of those respondents who reported that their units provided written communication, ICU administrators more often reported that the written information included both a summary of treatments provided $(43 \%$ vs. $9 \%, p=0.017)$ and treatment plans $(83 \%$ vs. $23 \%, p<0.001)$, and less commonly reported the written information to include information about the hospital ward the patient was transferring to $(43 \%$ vs. $83 \%, p=0.005)$, when compared to ward administrators.

Table 2 Number (\%) of Respondents Identifying Opportunities for Improvement in the Transition of Patient Care from the Intensive Care Unit to Hospital Ward

\begin{tabular}{|c|c|c|c|}
\hline Areas for improvement & $\begin{array}{l}\operatorname{ICU}(\%) \\
(n=108)\end{array}$ & $\begin{array}{l}\text { Ward (\%) } \\
(n=160)\end{array}$ & $\begin{array}{l}p \\
\text { value }\end{array}$ \\
\hline $\begin{array}{l}\text { Information provided to } \\
\text { patient and family }\end{array}$ & $64(67)$ & $95(73)$ & 0.297 \\
\hline $\begin{array}{l}\text { Patient and family } \\
\text { participation during transfer }\end{array}$ & $66(69)$ & $84(65)$ & 0.515 \\
\hline Collaboration between units & $62(65)$ & $74(57)$ & 0.245 \\
\hline Continuity of care & $62(65)$ & $71(55)$ & 0.132 \\
\hline Completeness of information & $56(58)$ & 77 (59) & 0.892 \\
\hline $\begin{array}{l}\text { Timely transfer of } \\
\text { information }\end{array}$ & $51(53)$ & $58(45)$ & 0.206 \\
\hline Accuracy of information & $33(34)$ & $50(38)$ & 0.529 \\
\hline Access to information & $27(28)$ & $31(24)$ & 0.467 \\
\hline Duplication of information & $25(26)$ & $25(19)$ & 0.223 \\
\hline
\end{tabular}

Data are expressed as number (percentage) unless otherwise indicated Response rates for individual questions were $89 \%$ for ICUs and $88 \%$ for wards

Responses are not mutually exclusive 
Opportunities for Improvement. Table 2 outlines Administrators' perspectives on potential opportunities for improving the ICU-to-ward transfer process. The three most frequently reported opportunities for improvement were the same for both administrator groups and included the amount or type of information provided to patients and families, patient and family participation during transfer, and collaboration between units.

Subgroup Analyses. The patterns of administrator survey responses were broadly similar when stratified according to profession (i.e., medical and nursing). Ward administrator perspectives were similar whether they managed a medical or surgical unit.

\section{Content Analysis of Transfer Tools}

The 268 survey participants provided 108 transfer tools from 53 hospitals. Of these, 48 tools (from 29 hospitals) satisfied the inclusion criteria, of which 21 unique transfer tools were included in the content analysis (Table 3). The 60 tools excluded from analysis were not related to the transfer process (42\%), facilitated hospital to community transfer (35\%), a transfer order set $(22 \%)$, or medication reconciliation instruments (3\%). Among the tools included in the analyses, the most common purpose was transfer of pertinent clinical information between providers $(71 \%)$ and/or to provide a checklist for provider tasks $(38 \%)$. The majority of tools were paperbased $(86 \%)$ and contained both forced-choice $(95 \%)$ and open-text $(57 \%)$ options for populating information. Almost half of the tools did not specify the sending provider role (48\%), receiving provider role $(52 \%)$, or the target patient population $(88 \%)$. The content analysis identified six broad categories of information elements captured within the tools:

Table 3 Tools to Facilitate Transfer of Care from the ICU to Hospital Ward

\begin{tabular}{ll}
\hline \hline Characteristic & No. (\%) (n=21) \\
\hline Sending ICU provider & \\
Attending physician & $1(5)$ \\
Nurse & $9(43)$ \\
Respiratory therapist & $1(5)$ \\
Not specified & $10(48)$ \\
Ward recipient & $0(0)$ \\
Attending physician & $7(33)$ \\
Nurse & $1(5)$ \\
Respiratory therapist & $2(10)$ \\
Patient/family & $11(52)$ \\
Not specified & $15(71)$ \\
Purpose of tool* & $8(38)$ \\
Transfer of pertinent clinical data & $2(10)$ \\
Provider checklist of required tasks & \\
Information brochure for patient/family & $18(86)$ \\
Medium of tool & $3(14)$ \\
Paper & \\
Electronic & $20(95)$ \\
Format of tool* & $12(57)$ \\
Forced-choice responses & \\
Open-text responses &
\end{tabular}

*Responses are not mutually exclusive demographics, patient clinical course, corrective aids, mobility at discharge, review of systems, documentation of transfer procedures. Overall, $95 \%$ of the tools contained information elements from one or more of the categories, with $14 \%$ of tools containing elements from all six categories.

Demographics. Supplementary Table 3 (available online) describes the distinct information elements that were identified within the category of demographics $(76 \%)$. Of the tools that contained elements related to patient demographics, 76\% included basic elements (e.g., addressograph [43\%], primary language spoken [24\%]), 19\% included legal elements (e.g., next of kin [19\%]), and $14 \%$ included holistic/family elements (e.g., psychosocial needs [5\%]).

Patient Clinical Course. Tools containing information elements related to patient clinical course $(76 \%)$ are outlined in Table 4. Related subcategories included diagnoses/operative procedures related to present illness $(62 \%)$ (e.g., primary diagnosis [57\%]), investigations/operative procedures during ICU stay (38\%), ongoing issues/problems at times of ICU transfer (57\%) (e.g., concerns/comments [43\%]), medications (14\%) (e.g., active medications on transfer [14\%]), and past illness (57\%) (e.g., allergies [52\%]).

Corrective Aids. Supplementary Table 4 (available online) outlines information elements related to corrective aids (19\%). Subcategories within corrective aids included such aids as vision (14\%), hearing (14\%), and dentures (14\%).

Mobility at Discharge. Elements related to mobility at time of transfer $(57 \%)$ are described in Supplementary Table 5 (available online). The most commonly included information elements were fall risk assessment (48\%), transfer with assist

Table 4 ICU Transfer Tool Information Elements Pertaining to Patient Clinical Course

\begin{tabular}{ll}
\hline \hline Element & $\begin{array}{l}\text { No. (\%) } \\
(\boldsymbol{n}=\mathbf{2 1 )}\end{array}$ \\
\hline Patient clinical course & $16(76)$ \\
Diagnoses/operative procedures related to present illness & $13(62)$ \\
Primary diagnosis & $12(57)$ \\
Secondary diagnoses & $2(10)$ \\
Investigations/operative procedures during ICU stay & $8(38)$ \\
Ongoing issues/problems at time of ICU transfer & $12(57)$ \\
Concerns/comments & $9(43)$ \\
Abnormal labs & $3(14)$ \\
Consults & $4(19)$ \\
Medications & $3(14)$ \\
Active medications on transfer & $3(14)$ \\
Home medications & $1(5)$ \\
Past illness & $12(57)$ \\
Allergies & $11(52)$ \\
Past medical/surgical history & $7(33)$ \\
Substance dependency issues & $3(14)$ \\
Immunizations & $1(5)$ \\
Comorbidities & $1(5)$ \\
\hline
\end{tabular}

Categories and items are not mutually exclusive 
Table 5 ICU Transfer Tool Information Elements Pertaining to Review of Systems

\begin{tabular}{|c|c|}
\hline Elements & $\begin{array}{l}\text { No. }(\%)(n= \\
21)\end{array}$ \\
\hline Review of systems & $15(71)$ \\
\hline Infectious disease & $10(48)$ \\
\hline Isolation/precautions status & $10(48)$ \\
\hline Airborne & $1(5)$ \\
\hline Contact & $2(5)$ \\
\hline Droplet & $2(5)$ \\
\hline Colonization/infection status & $6(29)$ \\
\hline Methicillin-resistant Staphylococcus aureus & $2(10)$ \\
\hline \multicolumn{2}{|l|}{ (MRSA) positive } \\
\hline Vancomycin-resistant Enterococcus (VRE) positive & $2(10)$ \\
\hline Clostridium difficile $(C$. diff) positive & $1(5)$ \\
\hline Date/time of swab & $1(5)$ \\
\hline Neurological & $13(62)$ \\
\hline Signs and symptoms & $10(48)$ \\
\hline Confusion & $7(33)$ \\
\hline Agitation & $5(24)$ \\
\hline Dysphagia & $2(10)$ \\
\hline Delirium & $3(14)$ \\
\hline Level of consciousness & $10(48)$ \\
\hline Glasgow Coma Scale & $8(38)$ \\
\hline Oriented & $5(24)$ \\
\hline Cooperative & $1(5)$ \\
\hline Pupillary assessment & $2(10)$ \\
\hline Pain assessment & $8(38)$ \\
\hline Pain management & $7(33)$ \\
\hline Intravenous or oral analgesics & $5(24)$ \\
\hline Restraints & $2(10)$ \\
\hline Mechanical & $2(10)$ \\
\hline Pharmacological & $1(5)$ \\
\hline Psychiatric & $5(24)$ \\
\hline Need for observation (close/constant) & $5(24)$ \\
\hline Cardiovascular & $13(62)$ \\
\hline Vitals & $11(52)$ \\
\hline Rhythm & $4(19)$ \\
\hline Intravascular line management & $9(43)$ \\
\hline Art line removal date/time & $3(14)$ \\
\hline CVC removal date/time & $1(5)$ \\
\hline Respiratory & $15(71)$ \\
\hline Respiratory vital signs & $12(57)$ \\
\hline Oxygen $\left(\mathrm{O}_{2}\right)$ delivery rate and method & $12(57)$ \\
\hline Respiratory Rate & $3(14)$ \\
\hline Peripheral capillary oxygen saturation currently & $3(14)$ \\
\hline Current fraction of inspired oxygen $\left(\mathrm{FiO}_{2}\right) /$ peripheral & $2(10)$ \\
\hline \multicolumn{2}{|l|}{ capillary oxygen saturation $\left(\mathrm{SpO}_{2}\right)$ goals } \\
\hline Symptoms & $2(10)$ \\
\hline Dyspnea & $2(10)$ \\
\hline Orthopnea & $2(10)$ \\
\hline Cough strength & $1(5)$ \\
\hline Airway concerns & $7(33)$ \\
\hline Breath/chest sounds & $5(24)$ \\
\hline Bilevel positive airway pressure (BiPAP)/continuous & $3(14)$ \\
\hline \multicolumn{2}{|l|}{ positive airway pressure (CPAP) } \\
\hline Concerns/difficult airway & $2(10)$ \\
\hline Chest tubes & $7(33)$ \\
\hline Tracheostomy & $9(43)$ \\
\hline Tracheostomy insertion date & $7(33)$ \\
\hline Tracheostomy size & $6(29)$ \\
\hline Type of tracheostomy & $5(24)$ \\
\hline Extubation date/time & $4(19)$ \\
\hline Cuff inflated & $2(10)$ \\
\hline Suction passes in last $24 \mathrm{~h}$ & 4 (19) \\
\hline Number of days intubated & $2(10)$ \\
\hline Endocrine & $5(24)$ \\
\hline Sliding scale insulin & 3 (14) \\
\hline Gastrointestinal & $13(62)$ \\
\hline Signs and symptoms & $3(14)$ \\
\hline Abdominal distension & $3(14)$ \\
\hline Vomiting & $3(14)$ \\
\hline Nausea & $3(14)$ \\
\hline Appetite change & $2(10)$ \\
\hline Stoma assessment & $1(5)$ \\
\hline Signs of gastrointestinal bleeding & $1(5)$ \\
\hline Function & $12(57)$ \\
\hline Last bowel movement & $10(48)$ \\
\hline
\end{tabular}

Table 5. (continued)

\begin{tabular}{|c|c|}
\hline$\overline{\text { Elements }}$ & $\begin{array}{l}\text { No. (\%) } \\
(n=21)\end{array}$ \\
\hline Diet & $9(43)$ \\
\hline Diarrhea/loose & $3(14)$ \\
\hline Continent or incontinent & $2(10)$ \\
\hline Feeding or decompression tubes & $11(52)$ \\
\hline Nasogastric, nasojejunal, or silastic tube & $11(52)$ \\
\hline Location of nasogastric or nasojejunal tube & $1(5)$ \\
\hline Genitourinary & $13(62)$ \\
\hline Signs and symptoms & $4(19)$ \\
\hline Incontinent & $4(19)$ \\
\hline Dysuria & $2(10)$ \\
\hline Bleeding & $2(10)$ \\
\hline Foley and urine output & $11(52)$ \\
\hline Foley date/time inserted & $10(48)$ \\
\hline Current balance in/out & $4(19)$ \\
\hline Urine last $24 \mathrm{~h}$ & $3(14)$ \\
\hline Urine color & $3(14)$ \\
\hline Date last dialyzed & $2(10)$ \\
\hline Gynecology & $2(10)$ \\
\hline Pregnant & $2(10)$ \\
\hline Last menstrual period & $2(10)$ \\
\hline Integumentary and surgical wound/drain management & $14(67)$ \\
\hline Skin ulcer monitoring & $13(62)$ \\
\hline Skin assessment (color, condition, etc.) & $12(57)$ \\
\hline Braden score & $5(24)$ \\
\hline Pressure ulcer & $2(10)$ \\
\hline Surgical wound and drain assessment & $13(62)$ \\
\hline Wound care instructions & $12(57)$ \\
\hline Wound location & $7(33)$ \\
\hline Drains/tubes & $5(24)$ \\
\hline Next dressing change & $1(5)$ \\
\hline High-risk transfer criteria & $13(62)$ \\
\hline Heart rate $<40$ or $>140 \mathrm{bpm}$ & $1(5)$ \\
\hline
\end{tabular}

bmp, beats per minute; $\mathrm{mmHg}$, millimeters of mercury

Categories and items are not mutually exclusive

needed (29\%), and assistive devices required (chair, walker, cane) $(24 \%)$.

Review of Systems. Table 5 outlines information elements related to a review of systems $(71 \%)$. Elements were further organized into subcategories, of which respiratory $(71 \%)$ and integumentary and surgical wound/drain management (67\%) were included most often.

Documentation of Transfer Procedures. Table 6 summarizes the information elements contained within the tools that relate to the documentation of transfer procedures (86\%). Distinct information elements were subcategorized into reports attached to the transfer information (i.e., reports included with the patient chart on transfer) (14\%), information related to the transferring service (86\%), information related to the accepting service (57\%), information about the recording ICU provider $(76 \%)$, and information about the receiving ward provider $(48 \%)$.

\section{DISCUSSION}

This cross-sectional study explored the perspectives of ICU and ward administrators on current transfer of care practices from ICU to ward and the transfer tools used to facilitate this process. Our findings suggest the perspectives of ICU and 
Table 6 ICU Transfer Tool Information Elements Pertaining to Documentation of Transfer Procedures

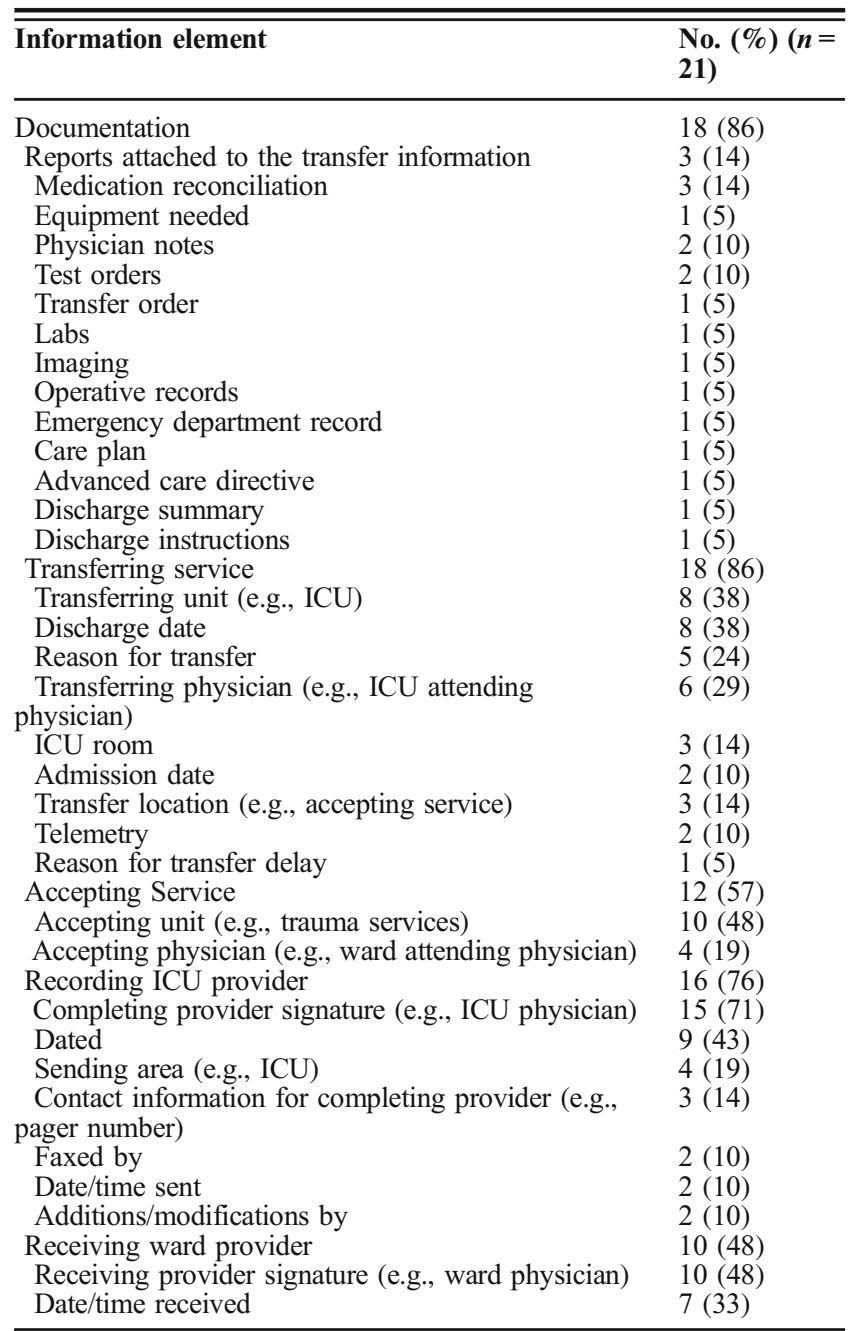

Categories and items are not mutually exclusive

ward administrators are broadly similar, supporting the notion that transfer of care is meant to be a "handoff" and both healthcare teams need to have a shared understanding of the process. ${ }^{14}$ The large number of diverse transfer tools available highlights the limited data available to guide the use tools to facilitate transfers of care. ${ }^{33}$

Our data highlight important discrepancies between administrator perceptions of the transfer process and the tools used to facilitate transitions of care. ICU administrator respondents perceived that discharge summaries are routinely included as part of the transfer procedure, in contrast to the perceptions of ward administrators. The majority of both ICU and ward administrator respondents reported that medication reconciliation is part of current ICU transfer practices, and yet only $14 \%$ of the transfer tools contained information elements related to this. These discrepancies suggest both a mismatch between what administrators feel should be included in the transfer process and the tools available to facilitate the process and a lack consensus around the essential elements that should be included. ${ }^{21,33}$ While tools should be tailored to the needs of local environments, ${ }^{34}$ the extensive variation contained within the tools used by the different institutions in our study does not make sense. Presumably, there is a core group of information elements and transfer procedures that should be consistent across organizations. Could a catalog of candidate essential information elements, such as the one resulting from this work, serve as the starting of a foundation for a "base model" transfer tool that organizations could then adapt to their settings? The results of the survey data and content analysis suggest the following key elements be considered for inclusion in a transfer summary: patient demographics and preexisting comorbidities, risk assessment (e.g., frailty status, severity of illness, goals of care), pertinent information of the patient's clinical course (e.g., primary diagnosis, active and resolved problems), pertinent elements related to review of systems (e.g., isolation status, intravascular devices, drains and catheters), and documentation related to the transfer process (e.g., checklist confirming verbal and written handoff, sending and receiving provider contact information).

Following a scoping review of the ICU transfer literature, Stelfox and colleagues ${ }^{6}$ suggest that a multi-pronged approach is likely necessary to "reengineer" the transfer process and highlight a need for better patient and family engagement within the transfer process. ${ }^{33,}{ }^{35} \mathrm{~A}$ recent qualitative study of perceived barriers and facilitators to high-quality transfer practices reported that ICU providers, ward providers, and patients and families all recommended the use of both communication tools (e.g., standardized written summary of patient course and treatment plan) and employing multimodal communication practices (i.e., verbal, written, electronic) to document the transfer to ensure continuity of care. ${ }^{36}$ This was further echoed in our study, with both administrator groups identifying an opportunity to improve transfer practices by providing patients and families with more information and engaging them in the transfer process (e.g., shared decision-making). This reflects growing efforts in healthcare to engage relevant stakeholders including patients in research, quality improvement, and healthcare service planning. ${ }^{37-40}$ That being said, a minority of ICU and ward administrators reported providing written information to patients and families and only $10 \%$ of the transfer tools were designed for a patient and family audience. The principles of human factors engineering provide a helpful framework that has been successfully applied to tool development in the field of medicine. ${ }^{41}$ These principles include early and consistent stakeholder engagement, development to fit to user needs, parsimony and tool order based on importance or chronological order of item use, subgrouping items into sections as required, evaluating usability and potential consequences (e.g., workflow impact) prior to implementation, pilot testing and validating the instrument before full implementation and scale-up, and updating the instrument as necessary with current best evidence or when system (operational) changes occur. ${ }^{41}$ Regardless of the framework used, the construction of any new decision-support tool should include 
rigorous validation efforts prior to implementation into patient care to ensure meaningful improvements to patient care and to avoid unforeseen consequences to workflow or quality of care as a result of sub-optimal patient transfer practices. ${ }^{42-44}$

There are four limitations that should be considered when interpreting these findings. First, we targeted only administrators because they are positioned to have broader institutionallevel perspectives that include workflow and multidisciplinary patient care practices. While the lens through which administrators view transfers of care is likely different than that of frontline providers (e.g., physicians, nurses), the perspectives were similar with those communicated by frontline providers, patients, and family members in other steps of our research program. ${ }^{8,36,45}$ Second, the two surveys were not distributed concurrently because the ward administrator sampling frame was derived from the survey responses of ICU administrators. We acknowledge that it is possible, although unlikely, that institutions may have substantially modified transfer polices during this 1-year time period. Third, the cross-sectional survey design used relies on self-report and is susceptible to social desirability bias. The survey responses may reflect idealized processes instead of actual occurrences. Our data provide an environmental scan of self-reported transfer practices and tools used to facilitate ICU transfers and highlight high variation across both. Finally, we restricted our study to Canadian acute care hospitals. The organization of healthcare varies between countries and the generalizability of the results is unknown.

\section{CONCLUSION}

The transfer of critically ill patients from the ICU to hospital ward is challenging and high risk. Our data indicate that ICU and ward administrators' perspectives on both the current ICU transfer practices and the suggested areas for improvement (e.g., increased meaningful patient engagement in patient transfer) are similar. The lack of consistency in the structure and content of ICU transfer tools highlights a need for the development and implementation of an evidence-informed ICU transfer tool designed to standardize key information elements between services, as a means to improve the quality of care during these vulnerable periods in healthcare delivery.

Corresponding Author: Henry Thomas Stelfox, MD, PhD; Departments of Critical Care Medicine, Medicine and Community Health Sciences, O'Brien Institute for Public Health, University of Calgary and Alberta Health Services - Calgary Zone, Calgary, Alberta, Canada (e-mail: tstelfox@ucalgary.ca).

Authors' Contributions JMB and HTS contributed to the study's conception and design. JMB collected and cleaned the data. JMB, $D J R, J P L$, and HTS performed and interpreted the analyses and assisted in the successive revisions of the final manuscript. All four authors read and approved the final manuscript.

Funding Information Henry T Stelfox was supported by a New Investigator Award from the Canadian Institutes of Health Research and a Population Health Investigator Award from Alberta Innovates Health Solutions. Funding sources had no role in the design of the study.

\section{Compliance with Ethical Standards:}

Ethics approval was obtained from the Conjoint Health Research Ethics Board at the University of Calgary (EID-24282).

Conflict of Interest: The authors declare that they do not have a conflict of interest.

Disclaimer: This work has not been published or presented elsewhere.

Abbreviations: ICU, intensive care unit

\section{REFERENCES}

1. Society of Critical Care Medicine. About critical care: team; 2017 Available from: http://www.myicucare.org/About-Critical-Care/Pages / Team.aspx. Accessed July 2, 2018.

2. Canadian Critical Care Society. For patients and families; 2014. Available from: http://www.canadiancriticalcare.org/Patients-\&-Families. Accessed July 2, 2018.

3. Tonelli MR, Misak CJ. Compromised autonomy and the seriously ill patient. Chest 2010;137(4):926-31.

4. Bell CM, Brener SS, Gunraj N, Association of ICU or hospital admission with unintentional discontinuation of medications for chronic diseases. JAMA 2011;306(8):840-7.

5. Garrouste-Orgeas M, Timsit JF, Vesin A, et al. Selected medical errors in the intensive care unit: results of the IATROREF study: parts I and II. Am J Respir Crit Care Med 2010;181(2):134-42.

6. Stelfox HT, Lane D, Boyd JM, et al. A scoping review of patient discharge from intensive care: opportunities and tools to improve care. Chest 2015; 147(2):317-27.

7. Hosein FS, Roberts DJ, Turin TC, Zygun D, Ghali WA, Stelfox HT. A meta-analysis to derive literature-based benchmarks for readmission and hospital mortality after patient discharge from intensive care. Crit Care 2014;18(6):715.

8. Li P, Boyd JM, Ghali WA, Stelfox HT. Stakeholder views regarding patient discharge from intensive care: suboptimal quality and opportunities for improvement. Can Respir J 2015;22(2):109-18.

9. Haggstrom M, Backstrom B. Organizing safe transitions from intensive care. Nurs Res Pract 2014;2014:175314.

10. Bagshaw SM, Opgenorth D, Potestio M, et al. Healthcare provider perceptions of causes and consequences of ICU capacity strain in a large publicly funded integrated health region: a qualitative study. Crit Care Med 2017;45(4):e347-56.

11. Stelfox HT, Hemmelgarn BR, Bagshaw SM, et al. Intensive care unit bed availability and outcomes for hospitalized patients with sudden clinical deterioration. Arch Intern Med 2012;172(6):467-74.

12. Aiken LH, Clarke SP, Sloane DM, Sochalski J, Silber JH. Hospital nurse staffing and patient mortality, nurse burnout, and job dissatisfaction. JAMA 2002;288(16): 1987-93.

13. Australian Commission on Safety and Quality in Healthcare. Clinical handover and patient safety; 2005. Available from: https://www.safetyandquality.gov.au/wp-content/uploads/2012/01/clinhovrlitrev.pdf. Accessed July 2, 2018.

14. Patient Safety Network. Patient safety primers: handoffs and signouts; 2016. Available from: https://psnet.ahrq.gov/primers/primer/9. Accessed July 2, 2018.

15. Institute of Medicine Committee on the Quality of Health Care in America. Crossing the Quality Chasm: A New Health System for the 21st Century. Washington, DC: National Academic Press; 2001.

16. National Transitions of Care Coalition. Improving transitions of care; 2010. Available from: http://www.ntocc.org/portals/0/pdf/resources/ ntoccissuebriefs.pdf. Accessed July 2, 2018.

17. Stutzman SE, Olson DM, Greilich PE, Abdulkadir K, Rubin MA. The patient and family perioperative experience during transfer of care: a qualitative inquiry. AORN J 2017;105(2):193-202.

18. Li P, Stelfox HT, Ghali WA. A prospective observational study of physician handoff for intensive-care-unit-to-ward patient transfers. Am J Med 2011;124(9):860-7. 
19. Haggstrom M, Asplund $\mathbf{K}$, Kristiansen L. Important quality aspects in the transfer process. Int J Health Care Gual Assur 2014;27(2): 123-39.

20. Massey D, Aitken LM, Chaboyer W. What factors influence suboptimal ward care in the acutely ill ward patient? Intensive Crit Care Nurs 2009;25(4):169-80.

21. Haggstrom $\mathbf{M}$, Asplund $\mathbf{K}$, Kristiansen $\mathbf{L}$. Struggle with a gap between intensive care units and general wards. Int $J$ Qual Stud Health Well-being 2009;4: 181-92

22. Buchner DL, Bagshaw SM, Dodek P, et al. Prospective cohort study protocol to describe the transfer of patients from intensive care units to hospital wards. BMJ Open 2015;5(7):e007913.

23. Duriau VJ, Reger RK, Pfarrer MD. A content analysis of the content analysis literature in organization studies: research themes, data sources, and methodological refinements. Organ Res Methods 2007;10(1):5-34.

24. Bryman A. Social Research Methods. New York, NY: Oxford University Press; 2001.

25. Gawande A. The Checklist Manifesto: How to Get Things Right. Great Britain: Profile Books LTD; 2010.

26. Haynes AB, Weiser TG, Berry WR, et al. A surgical safety checklist to reduce morbidity and mortality in a global population. NEJM 2009;360(5):491-9.

27. Zack JE, Garrison T, Trovillion E, et al. Effect of an education program aimed at reducing the occurrence of ventilator-associated pneumonia. Crit Care Med 2002;30(11):2407-12.

28. Winters BD, Gurses AP, Lehmann H, Sexton JB, Rampersad CJ, Pronovost PJ. Clinical review: checklists - translating evidence into practice. Crit Care 2009;13(6):210.

29. Hsieh HF, Shannon SE. Three approaches to qualitative content analysis. Qual Health Res 2005; 15(9):1277-88.

30. Glaser BG. Theoretical Sensitivity: Advances in the Methodology of Grounded Theory. Mill Valley, CA: Sociology Press; 1978.

31. Miles MB, Huberman M. Qualitative Data Analysis, 2nd edn. Thousand Oaks, CA: Sage; 1994.

32. Pope C, Ziebland S, Mays N. Qualitative research in health care. Analysing qualitative data. BMJ 2000;320(7227):114-6.

33. Rochester-Eyeguokan CD, Pincus KJ, Patel RS, Reitz SJ. The current landscape of transitions of care practice models: a scoping review. Pharmacotherapy 2016;36(1):117-33.
34. Costa DK, Kahn JM. Organizing critical care for the 21st Century. JAMA. 2016;315(8):751-2.

35. Enger R, Andershed B. Nurses' experience of the transfer of ICU patients to general wards: a great responsibility and a huge challenge. J Clin Nurs 2018;27(1-2):e186-94

36. de Grood C, Parsons Leigh J, Bagshaw SM, et al. Patient, family, and provider experiences with transfers from intensive care unit to hospital ward: a multi-centre qualitative study. CMAJ 2018;190(22):E669-76.

37. McKenzie E, Potestio ML, Boyd JM, et al. Reconciling patient and provider priorities for improving the care of critically ill patients: a consensus method and qualitative analysis of decision making. Health Expect 2017;20(6): 1367-74.

38. Partridge $\mathbf{N}$, Scadding $\mathbf{J}$. The James Lind Alliance: patients and clinicians should jointly identify their priorities for clinical trials. Lancet 2004;364(9449): 1923-4.

39. Pollock A, St George B, Fenton M, Firkins L. Top ten research priorities relating to life after stroke. Lancet Neurol 2012;11(3):209.

40. Manafo E, Petermann L, Vandall-Walker V, Mason-Lai P. Patient and public engagement in priority setting: a systematic rapid review of the literature. PloS One 2018;13(3):e0193579.

41. Carayon P, Xie A, Kianfar SH. Human factors and ergonomics. In Making Health Care Safer II: An Updated Critical Analysis of the Evidence for Patient Safety Practices. Rockville, MD: Agency for Healthcare Research and Quality; 2013.

42. Treadwell JR, Lucas S, Tsou AY. Surgical checklists: a systematic review of impacts and implementation. BMJ Qual Saf 2014;23(4):299-318.

43. Hales B, Terblanche M, Fowler R, Sibbald W. Development of medical checklists for improved quality of patient care. Int $J$ Qual Health Care 2008;20(1):22-30.

44. Gagliardi AR, Straus SE, Shojania KG, Urbach DR. Multiple interacting factors influence adherence, and outcomes associated with surgical safety checklists: a qualitative study. PloS One 2014;9(9):e108585.

45. Stelfox HT, Leigh JP, Dodek PM, et al. A multi-center prospective cohort study of patient transfers from the intensive care unit to the hospital ward. Intensive Care Med 2017;43(10):1485-94. 